\title{
BMJ Open Understanding the priorities of residents, family members and care staff in residential aged care using $Q$ methodology: a study protocol
}

\author{
Kristiana Ludlow, ${ }^{\odot}$ Kate Churruca, Louise A Ellis, ${ }^{\circledR}$ Virginia Mumford, ${ }^{\odot}$ \\ Jeffrey Braithwaite
}

To cite: Ludlow K, Churruca K, Ellis LA, et al. Understanding the priorities of residents, family members and care staff in residential aged care using $Q$ methodology: a study protocol. BMJ Open 2019;9:e027479. doi:10.1136/ bmjopen-2018-027479

\section{- Prepublication history for} this paper is available online. To view these files, please visit the journal online (http://dx.doi org/10.1136/bmjopen-2018027479).

Received 24 October 2018 Revised 21 December 2018 Accepted 7 January 2019
Check for updates

(C) Author(s) (or their employer(s)) 2019. Re-use permitted under CC BY-NC. No commercial re-use. See rights and permissions. Published by BMJ.

Australian Institute of Health Innovation, Macquarie University, Sydney, New South Wales, Australia

Correspondence to Kristiana Ludlow; kristiana.ludlow@mq.edu.au

\section{ABSTRACT}

Introduction Residential aged care facilities (RACFs) are under increasing pressure to provide high-quality, round the clock care to consumers. However, they are often understaffed and without adequate skill mix and resources. As a result, staff must prioritise care by level of importance, potentially leading to care that is missed, delayed or omitted. To date, the literature on prioritisation and missed care has been dominated by studies involving nursing staff, thereby failing to take into account the complex networks of diverse stakeholders that RACFs comprise. This study aims to investigate the priorities of residents, family members and care staff in order to make comparisons between how care is prioritised in RACFs by the different stakeholder groups.

Methods and analysis This study comprises a $Q$ sorting activity using $Q$ methodology, a think-aloud task, a demographics questionnaire and semi-structured interview questions. The study will be conducted in five RACFs across NSW and QLD, Australia. Using purposive sampling, the project will recruit up to 33 participants from each of the three participant groups. Data from the $Q$ sorting activity will be analysed using the analytic software PQMethod to identify common factors (shared viewpoints). Data from the think-aloud task and semi-structured interviews questions will be thematically analysed using the Framework Method and NVivo qualitative data analysis software.

Ethics and dissemination The study has been approved by St Vincent's Health and Aged Care Human Research and Ethics Committee and Macquarie University Human Research Ethics Committee. It is expected that findings from the study will be disseminated: in peer-reviewed journals; as an executive report to participating facilities and a summary sheet to participants; as a thesis to fulfill the requirements of a Doctor of Philosophy; and presented at conferences and seminars.

\section{INTRODUCTION}

\section{Background}

One of the biggest challenges healthcare systems face globally is how to meet the care needs of ageing populations. ${ }^{12}$ Individuals over the age of 65 currently make up
Strengths and limitations of this study

- This study presents a novel approach to understanding prioritisation in residential aged care by comparing the priorities of residents, family members and care staff, in a research area primarily dominated by the study of clinical staff.

- A tailored Q methodology approach will enable residents who are often excluded from research to participate in this study, including residents with vision impairment, hearing loss, or mild cognitive impairment.

- Residents with moderate to severe cognitive impairment are excluded from participating in the study; however, family members and staff members who care for residents with cognitive decline will be invited to participate.

- Additional research is recommended to explore the priorities of other stakeholder groups not involved in this study, for example, visiting physicians, allied health professional or volunteers.

- It is expected that study findings can be used to guide improvement strategies at the organisation level and policy level to deliver care that is consumer-centred while taking into consideration the priorities and role challenges of key stakeholders.

$8.7 \%$ of the world's total population, with some countries having up to three times this number (Japan, 27\%; Germany, 21\%; Australia, $16 \%$; USA $15 \%) .{ }^{3}$ This age group is expected to almost double by $2050,{ }^{4}$ and triple for those aged $>80 .^{5}$ As population growth and life expectancy continue to increase, health systems are faced with the challenge of providing sustainable services to older consumers that are safe, high quality, holistic, consumer-centred and affordable for consumers, institutions and funders.

\section{Residential aged care}

Accompanying the demographic shift towards older populations, there is a projected 
increase in the prevalence of dementia and multimorbidity. ${ }^{67}$ This means that not only are people living longer, but they are also living with more complex physical and cognitive needs, as well as a greater dependency on others to provide assistance with basic needs (eg, showering, mobility or eating). Dependent older individuals may require full-time care that their family members are not always able to provide. ${ }^{8}$ This places particularly high demands on residential care services in terms of funding, physical resources (eg, beds and equipment) and human resources, including workforce and training.

Unlike other healthcare systems, such as acute or primary care, residential aged care facilities (RACFs) serve as a home for consumers, ${ }^{910}$ providing social care, spiritual care, meaningful activities and physical assistance, in addition to medical care. ${ }^{11}$ RACFs must deliver round the clock care in which care staff are available to meet residents' varied needs, as well as managing interruptions to routine care, such as unexpected illness or injury.

\section{Prioritising care}

Despite being high-dependency environments, RACFs often lack adequate human resources in terms of both staffing levels and skill mix. ${ }^{12} 13$ These shortages and consequent time constraints mean that staff must continually prioritise and re-prioritise the care they provide by adapting to the situation at hand. Prioritisation can be understood as 'putting first', implying that something that is important or urgent has priority over what is less important or less urgent'. ${ }^{14}$ Priority-setting, or prioritisation of care, requires care staff to rank care activities in a hierarchical fashion according to the level of importance, where some tasks are assigned a lower priority. The action by which a lower priority care task is decisively traded off in favour of a higher priority task is known as 'implicit rationing, ${ }^{15}$

Prioritisation of tasks can result in 'missed care', ${ }^{13}$ which is 'any aspect of required patient care that is omitted (either in part or in whole) or delayed' ${ }^{16}$ Essentially, it is care that has fallen through the cracks in a complex-often, pressurised-system. Across acute and residential care settings, missed care is associated with poorer consumer outcomes, such as urinary tract infections, patient falls, pressure ulcers, and reduced patient satisfaction and quality of care. ${ }^{17}$

\section{The priorities of key stakeholders in RACFs}

Research on prioritisation and missed care predominantly focuses on the priorities of healthcare professionals, particularly nursing staff. ${ }^{17-19}$ This narrow focus limits our understanding of prioritisation by emphasising a single viewpoint instead of acknowledging healthcare systems as complex networks made up of diverse stakeholders. In RACFs, these networks comprise residents, family members, direct care staff (eg, carers, registered nurses and management), domestic staff (eg, cleaners), allied health professionals (eg, speech pathologists and

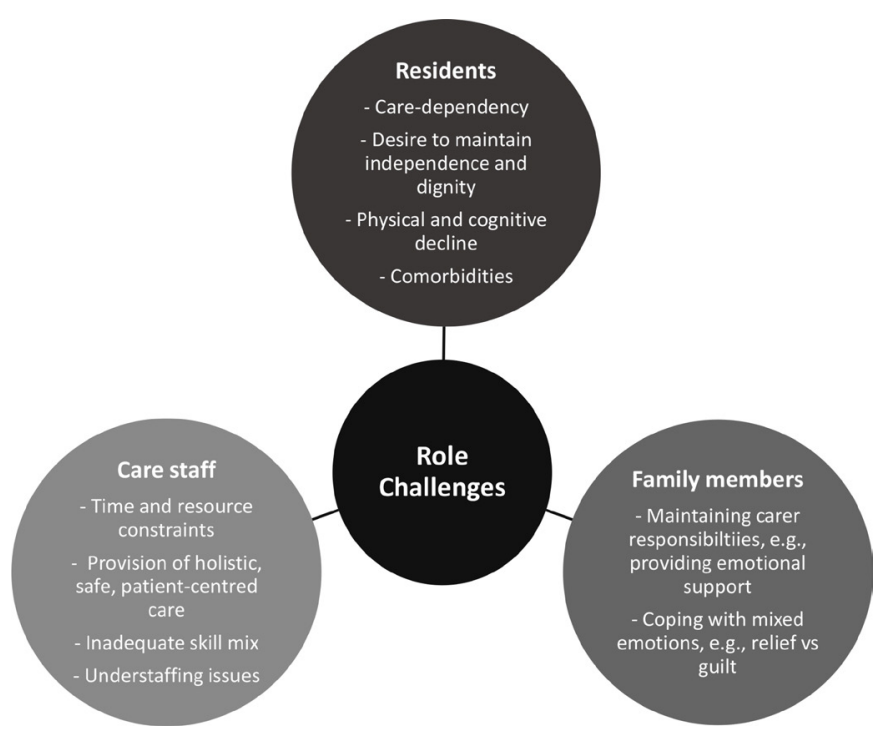

Figure 1 A selection of stakeholders' role challenges.

physiotherapists), visiting healthcare professionals (eg, physicians and dentists), volunteers, advocate groups and policy-makers.

Each stakeholder holds a distinct role, accompanied by different responsibilities and challenges, which can influence their priorities, that is, what is most important to them when it comes to care. For this proposed study, we focus on three central stakeholder groups: residents, family members and care staff. Figure 1 presents a selected summary of role challenges these stakeholders face, based on the literature. ${ }^{120-24}$

\section{Rationale}

The research team systematically reviewed the literature using the Preferred Reporting Items for Systematic Reviews and Meta-Analyses (PRISMA) framework ${ }^{25}$ to ascertain the current state of knowledge on 'unfinished care'-a collective term encompassing prioritisation, rationing, missed care, omitted care and care left undone. ${ }^{18}$ The majority of included studies examined the scope of the problem (eg, what care is missed, delayed or left undone) and antecedents (eg, what factors contribute to unfinished care, such as inadequate staffing or time constraints). ${ }^{1326-28}$ Few studies directly assessed prioritisation, ${ }^{29-31}$ demonstrating that there is limited knowledge about how care is prioritised in RACFs, particularly within the recent literature.

To deliver care that is more consumer-centred and takes the needs, as well as role challenges, of different stakeholders into consideration, it is important to obtain a deeper understanding of each groups' care priorities; what they value as most important in terms of care delivery. To the best of our knowledge, no other study has made comparisons between how care is prioritised by key stakeholder groups in residential aged care. The following objective and research questions were developed to address this gap. 


\section{Objective}

To investigate how care is prioritised by key stakeholders in RACFs and to make comparisons between the priorities of the different stakeholder groups.

\section{Research questions}

1. How do residents prioritise their care?

2. How do residents' family members prioritise care?

3. How do care staff prioritise the care provided to residents?

4. What are the differences and similarities between the priorities of the three stakeholder groups?

\section{Theoretical considerations}

The ways in which stakeholders prioritise care will be investigated through $\mathrm{Q}$ methodology. $\mathrm{Q}$ is a research method used to systematically study subjectivity and identify divergent viewpoints (factors), through the integration of quantitative and qualitative techniques. ${ }^{32}{ }^{33}$ While the $\mathrm{Q}$ approach is a method of doing research, in our work, we also draw on its theoretical underpinnings. In doing so, we make a number of assumptions. First, we assume that individuals are able to express their subjective views on what they value most (their priorities). Second, this subjectivity can be systematically collected and studied through $\mathrm{Q}$ methodology. ${ }^{33}$ Third, the number of distinctive viewpoints on a topic of interest is limited, known in the terminology of $Q$ as 'finite diversity'. ${ }^{35}$ Here, we assume that based on our use of $Q$, individuals' priorities can be examined to identify distinct, cohesive viewpoints shared by a number of participants.

In exploring the priorities of different stakeholders and making comparisons between them, we are also adopting a complexity lens. ${ }^{36}$ Through this lens, we view RACFs as complex systems, comprising diverse agents who interact and sense-make (interpret the world) in ways that create shared meanings. ${ }^{37-39}$ For this study, the portions of shared meaning under investigation are stakeholders' priorities. In a complex system, these collective interpretations are considered the basis for agents' interactions, which may, in turn, produce broader system-level behaviours, ${ }^{37}{ }^{40}$ for example, staff members may adapt to environmental constraints such as inadequate time or staff shortages by delaying or omitting aspects of care that are given lower priority.

While these broad theoretical and methodological principles guided our choice of method, we have avoided, at this stage, selecting a mid-range theory related to prioritisation, decision-making or care delivery in aged care. Q methodology is particularly suited to exploratory research, and it is then a convention to return to established theory to interpret results ${ }^{35}$; this will be the case for the proposed study, to understand the types of priorities participants have.

\section{METHODS AND ANALYSIS \\ Study design}

To answer the research questions, individual interviews will be carried out consisting of a $Q$ sorting activity using
Q methodology, ${ }^{32}$ a think-aloud task, ${ }^{41} 42$ a demographics questionnaire and semi-structured interview questions. ${ }^{43}$ The $\mathrm{Q}$ sorting activity will require participants to make prioritisation decisions by ordering aspects of care by degree of importance. This activity will allow for comparison of patients' sorting patterns, resulting in the identification of salient viewpoints, that is, holistic expressions of what matters most to participants in terms of the care provided in RACFs. Q methodology is a method that has been successfully used in studies of care staff, residents, residents' family members, and individuals with early stages of dementia, ${ }^{44-46}$ as well as in studies on prioritisation and priority setting. ${ }^{47} 48$

The $\mathrm{Q}$ sorting activity will be complemented by a concurrent think-aloud task and post-sort questions in order to provide insight into participants' thought processes and decision-making, for example, how they make prioritisation decisions and why certain aspects of care are viewed as more or less important than other aspects of care. Semi-structured interviews will provide a deeper understanding of prioritisation, for example, how participants perceive the priorities of other stakeholders, how care staff prioritise care during unexpected events or interruptions to care, and participants' experiences of unmet priorities.

\section{Sample and setting}

The research will be conducted in five RACFs across NSW and QLD, Australia. Purposive sampling in Q methodology allows for the capture of a diverse range of viewpoints on an issue that is relevant to participants ${ }^{48} 49$ and will be used to recruit participants from three groups: residents, family members and care staff.

$\mathrm{Q}$ methodology is concerned with identifying and understanding salient viewpoints on an issue, rather than generalising about the distribution of those viewpoints in the wider population. ${ }^{50}$ Therefore, unlike conventional survey techniques, large numbers of participants are not required nor recommended for $Q$ methodology. ${ }^{51}$ As a method, it privileges deep rather than broad data capture. Watts and Stenner suggest as a guideline that the number of participants (P set) should be less than the number of data items-referring to aspects of care in the proposed study. ${ }^{32}$ Participants will be recruited until data saturation is achieved, that is, when no new information is presenting ${ }^{52}$ or when the $\mathrm{P}$ set limit $(\mathrm{n}=33)$ for each group is reached.

\section{Inclusion criteria}

In order to participate in the study, participants must meet the following inclusion criteria: (1) be a current resident, family member of a resident or a staff member at a RACF; (2) have willingness and ability to provide informed consent; and (3) have the capacity to participate in an English-language interview. Additionally, resident participants can only be included in the study if their participation will not cause them any additional physical burden. Residents with mild cognitive impairment may participate 
in the study if they are able to give informed consent, as advised by facility management.

\section{Recruitment procedures}

Recruitment strategies for interviews were developed through discussions with managers of participating RACFs. Managers will identify residents, family members and care staff who meet the inclusion criteria. Potential enrollees will then be invited to participate in the study via invitation letters and Participant Information and Consent Forms (PICFs). Advertisement posters for family members will also be placed at the front desks of facilities, on notice boards and in activity rooms. The study commenced in August 2018 with an expected completion date of May 2020.

\section{Consent}

Consent will be obtained from all participants in written form or verbally if participants are unable to provide written consent. Due to the potential inclusion of residents with mild cognitive impairment, the proposed study will employ an ongoing consent process (process consent), in which consent is verbally re-confirmed throughout the study. ${ }^{53}$ Participants will be informed both verbally and within the PICFs that there is no obligation to participate in the study and that they can withdraw from the study at any time without consequence. If participants decide to withdraw during the study, they will be asked to sign a Withdrawal of Participation Form and no further personal information will be collected.

\section{Materials}

Demographics questionnaire

The demographics questionnaire has been designed to cover the following topics: age; gender; how long the participant has lived, worked or has had a family member living at the participating RACF; a self-reported singleitem measure of health (residents); job title (care staff).

\section{Q sort deck}

The $Q$ sorting activity will require participants to order aspects of care by level of importance using a set of cards ( $Q$ sort deck), each displaying three elements: a statement about an aspect of care; a visual representation of the corresponding statement and examples of the

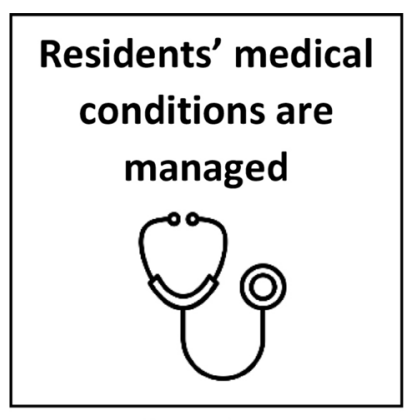

Front view

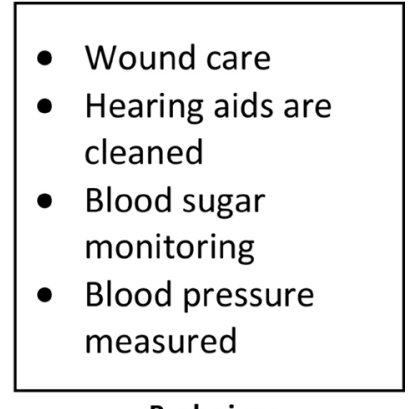

Back view
Figure 2 Example card: management of medical condition(s). care element (figure 2). The $\mathrm{Q}$ sort deck was developed through a five-stage process:

Stage 1: a comprehensive list of elements of care in RACFs, known as 'the concourse' was devised through a review of the literature. ${ }^{33}$

Stage 2: concept mind mapping ${ }^{54}$ was used to reduce the concourse to a subsample of items known as the $\mathrm{Q}$ set. ${ }^{55} \mathrm{~A}$ smaller $\mathrm{Q}$ set $(\mathrm{n}=34)$ will be used in this study in order to reduce the cognitive demand placed on resident participants, while adequately covering the range of care provided in RACFs.

Stage 3: Q set items were modified into statements suitable for ranking by each participant group, for example, My medical conditions are managed (resident statement); My family member's medical conditions are managed (family statement); and Residents' medical conditions are managed (care staff statement).

Stage 4: the $Q$ set was validated by three members of the managerial team from one of the participating sites to ensure that key aspects of care had been covered and that the language was appropriate and relevant for participants.

Stage 5: the Q set was transformed into a physical Q sort deck comprising a set of magnetic cards.

\section{Q sort diagram}

Cards will be sorted using a pre-established grid ( $Q$ sort diagram), which comprises a quasi-normal forced distribution with a rating scale from -4 (Least Important) to +4 (Most Important) (see figure 3). The $\mathrm{Q}$ sorting activity will be conducted on a whiteboard with the $Q$ sort diagram transposed on, allowing for easy attachment of magnetised cards.

\section{Semi-structured interview questions}

Semi-structured interviews will be tailored to each participant group and will address the following questions:

- What influences participants' priorities?

- How are participants' priorities communicated?

- How do priorities compare between different participant groups (eg, how do residents' priorities compare with the priorities of care staff)?

- What happens when priorities are not met?

- How do care staff manage their priorities when delivering care?

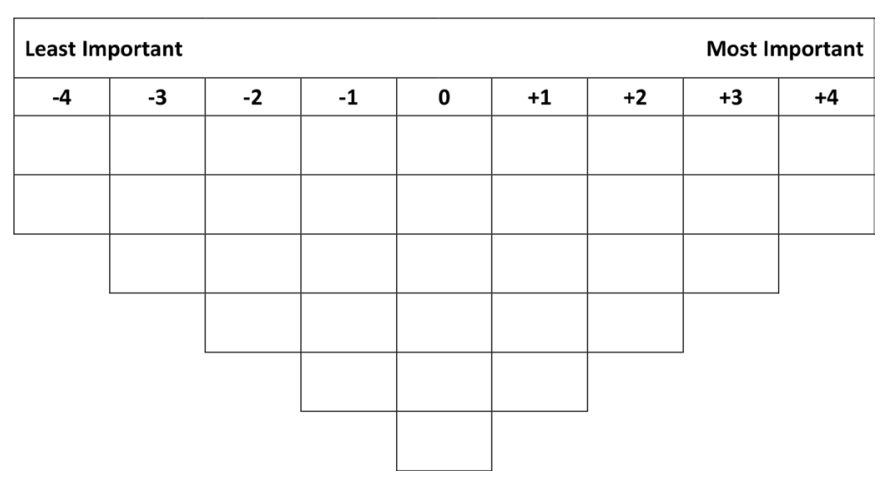

Figure $3 \mathrm{Q}$ sort diagram. 
- What challenges prevent care staff from meeting their priorities when delivering care?

- Is there any further information about priorities that participants want to discuss?

\section{Data collection}

$Q$ sorting activity and think-aloud task

Participants will be guided through the $Q$ sorting activity using established $Q$ methodology techniques. ${ }^{56}$ This will involve sorting the $\mathrm{Q}$ sort deck onto the $\mathrm{Q}$ sort diagram using the following condition of instruction: order the cards from 'least important' to 'most important' in terms of the care provided to you (residents); your family member (family members); or residents (care staff).

Concurrent to the $\mathrm{Q}$ sorting activity, participants will be asked to engage in a think-aloud task, ${ }^{41}{ }^{42}$ where they will be prompted by a member of the researcher team to vocalise their thoughts as they sort the cards. ${ }^{56}$ The thinkaloud task was piloted with an initial group of participants who found value in the opportunity to vocalise and explain their decisions. This task is a personalised one in which participants can engage to the degree to which they are willing and able. On completion of the $Q$ sorting activity, participants will be asked a series of open-ended questions about the way they sorted the cards ( $Q$ sorting). Post-Q sorting interviews will assist in the interpretation of individual Q sorts as well as the interpretation of shared viewpoints across participants. ${ }^{51}$ Questions will focus on the placement of salient cards and the decision-making process, and will build on responses from the think-aloud task.

\section{Demographics questionnaire and semi-structured interview questions}

Following the $\mathrm{Q}$ sorting activity, participants will be administered the demographics questionnaire. They will be given the option to complete the semi-structured interviews immediately after the demographics questionnaire or at another time if they prefer. The $\mathbf{Q}$ sorting activity, think-aloud task, demographics questionnaire and semi-structured interviews will be audio-recorded with participants' consent. A member of the research team will take field notes during interviews noting the context, participant mood, non-verbal behaviours, pace of decision-making and any interruptions to data collection. The audio recordings and field notes will ensure that participants' responses are accurately captured.

\section{Alternative administration}

Participants unable to attend on-site data collection will be offered an alternative method of study administration in which the demographics questionnaire and $Q$ sorting activity are administered via VQMethod, an online tool that mirrors physical card sorting. ${ }^{57}$ The semi-structured interview section of the study will be offered via telephone.

\section{Patient and public involvement}

The study design and methods were formulated based on experience with resident participants from a previous study on person-centred care, conducted at one of the participating sites. The $\mathrm{Q}$ sort deck was validated by three members of the management team at one of the participating facilities in order to ensure that the cards were appropriate for residents, family and staff members and that the cards adequately covered the care provided by the organisation. The study was piloted by a convenience sample of family members who currently have, or previously had, a relative living in a RACF to provide feedback on the card images and terminology, the card sorting processes and the abilities of residents to perform the study tasks.

\section{Analysis}

NVivo qualitative data analysis software V.1 $12^{58}$ will be used to organise data and assist data analysis. Data from the think-aloud task and semi-structured interviews will be transcribed verbatim and thematically analysed using the Framework Method. ${ }^{5960}$

Data from the $Q$ sorting activity will be analysed using established procedures within $Q$ methodology, which are based on inverted factor analysis techniques. ${ }^{61}$ The analysis aims to identify similar patterns in how participants have sorted the cards (ie, have prioritised care). Q sorts (individuals' card sequences) will be analysed separately for each of the three participant groups in order to identify similar viewpoints within each group. ${ }^{62}$ PQMethod, a statistical software designed for $Q$ methodology studies, ${ }^{63}$ will be used to identify highly correlated $Q$ sorts, known as 'factors', ${ }^{62} 64$ and 'factor arrays', which represent a single $Q$ sort characterising a 'best-estimate' of a factor. ${ }^{64}$ Factor arrays will be used in the interpretation process to produce meaningful narratives of different viewpoints on care priorities for each of the identified factors. ${ }^{65} \mathrm{~A}$ second-order factor analysis will then be performed in which the factor arrays from each of the three analyses will be entered into PQMethod as new $Q$ sorts. ${ }^{66}$ This process will enable a comparison between the priorities of residents, family members and care staff.

\section{EXPECTED OUTCOMES AND SIGNIFICANCE}

Prioritisation of care tasks can lead to care that is missed, omitted or delayed, ${ }^{16} 18$ and subsequently, adverse consequences for care consumers. For example, if regular repositioning or 'turning' is assigned a lower priority and is left undone, pressure ulcers may form, potentially resulting in infection and hospitalisation. ${ }^{17} 67$ Furthermore, if the priorities of care staff do not align with the priorities of residents and family members, then this may mean that what is valued most by residents and families is being overlooked.

Although we have some knowledge about what care is most often missed and what factors are associated with missed care, ${ }^{132628}$ less is known about how care is prioritised and what influences prioritisation decisions. This is especially true for RACFs, where unfinished care (prioritisation, rationing and missed care) is a research area that 
is fairly new and has been dominated by the experiences and perspectives of nursing staff. ${ }^{17-19}$

The proposed research aims to explore the phenomenon from different vantage points, providing a novel approach to the study of prioritisation. To the best of our knowledge, this will be the first study to compare how care is prioritised by key stakeholders (residents, family members and care staff) in RACFs. We expect this research to have valuable outcomes at the individual, organisation and system levels.

\section{Individual participants}

Although there are no guaranteed benefits to participating in the study, we anticipate that participants will find value in having the opportunity to express their opinions and reflect on their priorities. This study will also allow them insight into the priorities of other stakeholder groups, which may be information not otherwise easily accessed.

\section{Participating facilities}

By identifying shared priorities, as well as any discrepancies between the priorities of the three stakeholder groups, this research will highlight areas of care that are a high priority across the board, as well as signifying aspects of care that could be improved. This information can be used by facilities to guide their efforts of providing care which is in line with the priorities of different stakeholders.

Although missed care is not the direct focus of this study, it is a concept closely associated with prioritisation, and it is expected that this study will reveal information about unmet priorities, incidences of missed care and some of the challenges care staff experience when delivering care. This information may be used by RACFs to develop strategies aimed at reducing rates of missed care.

\section{Residential aged care policy}

The findings from this study may inform aged care policy about what key stakeholder groups value most in terms of care provision. This information is relevant to the aims of current healthcare reforms in which policy-makers are attempting to move away from traditional, medically focused models to more holistic and consumer-centred ones. ${ }^{68} 69$

\section{ETHICS AND DISSEMINATION}

This research project has been developed in accordance with the National Health and Medical Research Council (NHMRC) National Statement on Ethical Conduct in Research $^{70}$ and is approved by St Vincent's Health and Aged Care Human Research and Ethics Committee and Macquarie University Human Research Ethics Committee.

All data records will be de-identified using participant identification numbers. All digital copies of study materials, interview transcripts, field notes and audio recordings will be securely stored in electronic format on a password-protected database at Macquarie University.
Data will be stored for 5 years after the date of any publication resulting from this project, when it will then be disposed of in accordance with the requirements of the Macquarie University Code for the Responsible Conduct of Research.

De-identified research findings will be presented as an executive report to participating facilities and as summary sheets to participants. The research will be published as a thesis to fulfil the requirements of a Doctor of Philosophy. It is also intended that this research will be published in peer-reviewed journals and presented at national and international conferences and seminars. Any publication resulting from the findings will be de-identified to protect the privacy of participants.

Acknowledgements The authors would like to thank the members of the management team who reviewed the materials for the $Q$ sorting activity. They would also like to thank the individuals who piloted the study and provided their valuable feedback.

Contributors $\mathrm{KL}$ : conceptualised the study and produced the first draft of the manuscript. KL, KC, LAE, VM and JB: development of the study design. KL, KC, LAE and VM: devised the interview questions and the $Q$ set. All authors contributed to revisions of subsequent drafts of the manuscript and approved the final submission.

Funding This work is supported by a Macquarie University Research Training Program (MQRTP) Scholarship associated with the Australian Institute of Health project: Exploring complexity to create a sustainable healthcare system, as part of the National Health and Medical Research Council Partnership Centre grant in Health System Sustainability (ID:9100002).

Competing interests None declared.

Patient consent for publication Not required.

Ethics approval St Vincent's Health and Aged Care Human Research Ethics Committee, ID number: 18/18; and Macquarie University Human Research Ethics Committee.

Provenance and peer review Not commissioned; externally peer reviewed.

Open access This is an open access article distributed in accordance with the Creative Commons Attribution Non Commercial (CC BY-NC 4.0) license, which permits others to distribute, remix, adapt, build upon this work non-commercially, and license their derivative works on different terms, provided the original work is properly cited, appropriate credit is given, any changes made indicated, and the use is non-commercial. See: http://creativecommons.org/licenses/by-nc/4.0/.

\section{REFERENCES}

1. Amalberti R, Vincent C, Nicklin W, et al. Coping with more people with more illness. Part 1: the nature of the challenge and the implications for safety and quality. Int J Qual Health Care 2018.

2. Braithwaite J, Vincent C, Nicklin W, et al. Coping with more people with more illness. Part 2: new generation of standards for enabling healthcare system transformation and sustainability. Int J Qual Health Care 2018.

3. World Bank. Population ages 65 and above (\% of total). Washington, DC: World Bank, 2017. https://data.worldbank.org/indicator/SP.POP. 65UP.TO.ZS. (accessed 8 Oct 2018).

4. He W, Goodkind D, Kowa P, et al. An Aging World: 2015. International Population Reports. Washington, DC: U.S. Government Publishing Office, 2016. https://www.census.gov/content/dam/Census/library/ publications/2016/demo/p95-16-1.pdf. (accessed 8 Oct 2018).

5. Department of Economic and Social Affairs. World Population Ageing. Highlights. New York, NY: United Nations, 2015. http://www. un.org/en/development/desa/population/publications/pdf/ageing/ WPA2015_Highlights.pdf. (accessed 8 Oct 2018).

6. Kingston $A$, Robinson $L$, Booth $\mathrm{H}$, et al. Projections of multimorbidity in the older population in England to 2035: estimates from the Population Ageing and Care Simulation (PACSim) model. Age Ageing 2018;47:374-80.

7. Prince M, Comas-Herrera A, Knapp M, et al. The World Alzheimer Report 2016. Improving healthcare for people living with dementia: 
Coverage, quality and costs now and in the future. London, UK Alzheimer's Disease International, 2016. https://www.alz.co.uk/ research/WorldAlzheimerReport2016.pdf. (accessed 8 Oct 2018).

8. Ryan AA, Scullion HF. Nursing home placement: an exploration of the experiences of family carers. J Adv Nurs 2000;32:1187-95.

9. Hauge $\mathrm{S}$, Kristin $\mathrm{H}$. The nursing home as a home: a field study of residents' daily life in the common living rooms. J Clin Nurs 2008;17:460-7.

10. Nakrem S, Vinsnes AG, Harkless GE, et al. Ambiguities: residents' experience of 'nursing home as my home'. Int J Older People Nurs 2013;8:216-25.

11. Van Haitsma K, Curyto K, Spector A, et al. The preferences for everyday living inventory: scale development and description of psychosocial preferences responses in community-dwelling elders. Gerontologist 2013;53:582-95.

12. Willis E, Price K, Bonner R, et al. National aged care staffing and skills mix project report 2016. Meeting residents' care needs: A study of the requirement for nursing and personal care staff. Melbourne, Australia: Australian Nursing and Midwifery Federation, 2016. http:// www.anmf.org.au/documents/reports/National_Aged_Care_Staffing_ Skills_Mix_Project_Report_2016.pdf. (accessed 8 Oct 2018).

13. Henderson $\mathrm{J}$, Willis $\mathrm{E}$, Xiao $\mathrm{L}$, et al. Missed care in residential aged care in Australia: an exploratory study. Collegian 2017;24:411-6.

14. Werntoft E, Hallberg IR, Edberg AK. Prioritization and resource allocation in health care: the views of older people receiving continuous public care and service. Health Expect 2007;10:117-28.

15. Schubert M, Glass TR, Clarke SP, et al. Validation of the Basel Extent of Rationing of Nursing Care instrument. Nurs Res 2007:56:416-24.

16. Kalisch BJ, Landstrom GL, Hinshaw AS. Missed nursing care: a concept analysis. J Adv Nurs 2009;65:1509-17.

17. Recio-Saucedo A, Dall'Ora C, Maruotti A, et al. What impact does nursing care left undone have on patient outcomes? Review of the literature. J Clin Nurs 2018;27(11-12):2248-59.

18. Jones TL, Hamilton P, Murry N. Unfinished nursing care, missed care, and implicitly rationed care: State of the science review. Int $J$ Nurs Stud 2015;52:1121-37.

19. Hendry C, Walker A. Priority setting in clinical nursing practice: literature review. J Adv Nurs 2004;47:427-36.

20. Australian Institute of Health and Welfare. People's care needs in aged care. Canberra, Australia: AlHW, 2018. https://genagedcaredata.gov.au/Topics/Care-needs-in-aged-care. (accessed 8 Oct 2018).

21. Hall S, Dodd RH, Higginson IJ. Maintaining dignity for residents of care homes: a qualitative study of the views of care home staff, community nurses, residents and their families. Geriatr Nurs 2014;35:55-60.

22. Gaugler JE. Family involvement in residential long-term care: a synthesis and critical review. Aging Ment Health 2005;9:105-18.

23. Ryan AA, Scullion HF. Family and staff perceptions of the role of families in nursing homes. J Adv Nurs 2000;32:626-34.

24. Graneheim UH, Johansson A, Lindgren BM. Family caregivers' experiences of relinquishing the care of a person with dementia to a nursing home: insights from a meta-ethnographic study. Scand J Caring Sci 2014:28:215-24.

25. Moher D, Liberati A, Tetzlaff J, et al. Preferred reporting items for systematic reviews and meta-analyses: the PRISMA statement. Ann Intern Med 2009;151:264-9.

26. Knopp-Sihota JA, Niehaus L, Squires JE, et al. Factors associated with rushed and missed resident care in western Canadian nursing homes: a cross-sectional survey of health care aides. J Clin Nurs 2015;24(19-20):2815-25

27. Simmons SF, Durkin DW, Rahman AN, et al. Resident characteristics related to the lack of morning care provision in long-term care. Gerontologist 2013;53:151-61.

28. Zúñiga F, Ausserhofer D, Hamers JP, et al. The relationship of staffing and work environment with implicit rationing of nursing care in Swiss nursing homes-a cross-sectional study. Int J Nurs Stud 2015;52:1463-74.

29. Nortvedt P, Pedersen R, Grøthe KH, et al. Clinical prioritisations of healthcare for the aged-professional roles. $J$ Med Ethics 2008;34:332-5

30. Slettebø A, Kirkevold M, Andersen B, et al. Clinical prioritizations and contextual constraints in nursing homes-a qualitative study. Scand $J$ Caring Sci 2010;24:533-40.

31. Bowers BJ, Lauring $\mathrm{C}$, Jacobson $\mathrm{N}$. How nurses manage time and work in long-term care. J Adv Nurs 2001;33:484-91.

32. Watts S, Stenner P. Doing Q methodological research: theory, method and interpretation. Los Angeles, CA: SAGE Publications, 2012.

33. Brown S. A primer on $\mathrm{Q}$ methodology. Operant Subjectivity 1993;16:91-138.
34. Stephenson W. Introduction to Q-methodology. Operant Subjectivity 1993:17:1-13.

35. Stainton Rogers R. Q methodology. In: Smith JA, Harre R, Longenhove VI, eds. Rethinking methods in psychology. London, UK: Sage, 1995:178-93.

36. Anderson RA, Issel LM, McDaniel RR. Nursing homes as complex adaptive systems: relationship between management practice and resident outcomes. Nurs Res 2003;52:12-21.

37. Braithwaite J, Churruca K, Ellis LA, et al. Complexity science in healthcare - aspirations, approaches, applications and accomplishments: a white paper. Sydney, Australia: Australian Institute of Health Innovation, Macquarie University, 2017.

38. Lanham HJ, Leykum LK, Taylor BS, et al. How complexity science can inform scale-up and spread in health care: understanding the role of self-organization in variation across local contexts. Soc Sci Med 2013;93:194-202.

39. Weick KE. The social psychology of organizing. 2nd edn. New York, NY: McGraw-Hill, 1979.

40. Plsek PE, Greenhalgh T. The challenge of complexity in health care. BMJ 2001;323:625-8.

41. Charters $E$. The use of think-aloud methods in qualitative research an introduction to think-aloud methods. Brock Education Journal 2003;12:68-82.

42. Eccles DW, Arsal G. The think aloud method: what is it and how do I use it? Qual Res Sport Exerc Health 2017;9:514-31.

43. Low J. Unstructured and semi-structured interviews in health research. In: Saks M, Allsop J, eds. Researching health: qualitative, quantitative and mixed methods. 2nd ed. London, UK: SAGE, 2012:74-91.

44. Hill SR, Mason H, Poole M, et al. What is important at the end of life for people with dementia? The views of people with dementia and their carers. Int J Geriatr Psychiatry 2017;32:1037-45.

45. Lim SY, Chang SO. Nursing home staff members' subjective frames of reference on residents' achievement of ego integrity: A Q-methodology study. Jpn J Nurs Sci 2018;15:17-30.

46. White HK, Corazzini K, Twersky J, et al. Prioritizing culture change in nursing homes: perspectives of residents, staff, and family members. J Am Geriatr Soc 2012;60:525-31.

47. van Exel J, Baker R, Mason $\mathrm{H}$, et al. Public views on principles for health care priority setting: findings of a European cross-country study using Q methodology. Soc Sci Med 2015;126:128-37.

48. Alderson S, Foy R, Bryant L, et al. Using Q-methodology to guide the implementation of new healthcare policies. BMJ Qual Saf 2018;27:737-42.

49. Ramlo $S$. Mixed method lessons learned from 80 years of $Q$ Methodology. J Mix Methods Res 2016;10:28-45.

50. Watts S, Stenner P. Doing the fieldwork: participants, materials and procedure. In: Watts S, Stenner P, eds. Doing Q methodological research theory, method \& interpretation. London, UK: SAGE Publications, 2012:69-90

51. Watts $S$, Stenner P. Doing Q methodology: theory, method and interpretation. Qual Res Psychol 2005;2:67-91.

52. Glaser BG, Strauss AL. The discovery of grounded theory: strategies for qualitative research. New Brunswick, NJ: Aldine, 1967.

53. Murphy K, Jordan F, Hunter A, et al. Articulating the strategies for maximising the inclusion of people with dementia in qualitative research studies. Dementia 2015;14:800-24.

54. Davies M. Concept mapping, mind mapping and argument mapping: what are the differences and do they matter? High Educ 2011;62:279-301.

55. Paige JB, Morin KH. Q-sample construction: a critical step for a Q-methodological Study. West J Nurs Res 2016;38:96-110.

56. Innes K, Gillies K, Cotton S, et al. Q methodology with cognitive interviewing to rank the importance of informational items in a patient information leaflet. Trials 2015;16(S2):P76.

57. Nazariadli S. VQMethod. North Carolina State University, NC. 2018 https://www.vqmethod.com/ (accessed 8 Oct 2018).

58. QSR International Pty Ltd. NVivo qualitative data analysis Software. QSR International Pty Ltd; n.d. https://www.qsrinternational.com/ nvivo/what-is-nvivo (accessed 8 Oct 2018)

59. Ritchie J, Lewis J, eds. Qualitative research practice: a guide for social science students and researchers. London, UK: SAGE, 2003.

60. Gale NK, Heath G, Cameron E, et al. Using the framework method for the analysis of qualitative data in multi-disciplinary health research. BMC Med Res Methodol 2013;13:117.

61. Watts $S$, Stenner P. Introducing Q methodology: The inverted factor technique. In: Watts S, Stenner P, eds. Doing Q methodological research: theory, method and interpretation. London, UK: SAGE Publications, 2012:3-23.

62. Watts $S$, Stenner P. Understanding the analytic process (1): factor extraction. In: Watts S, Stenner P, eds. Doing Q methodological 
research theory, method \& interpretation. London, UK: SAGE Publications, 2012:91-112.

63. Schmolck P. The QMethod Page. 2018 http://schmolck.org/ qmethod/ (accessed 8 Oct 2018).

64. Watts S, Stenner P. Understanding the analytic process (2): factor rotation and the preparation of factor arrays. In: Watts $\mathrm{S}$, Stenner P, eds. Doing Q methodological research theory, method \& interpretation. London, UK: SAGE Publications, 2012:112-44.

65. Watts S, Stenner P. Factor interpretation. In: Watts S, Stenner P, eds. Doing $Q$ methodological research theory, method \& interpretation. London, UK: SAGE Publications, 2012:147-68.

66. Watts $S$, Stenner P. Basic design issues: research questions and $Q$ sets. In: Watts S, Stenner P, eds. Doing Q methodological research theory, method and interpretation. London, UK: SAGE Publications, 2012:49-68.
67. Schubert M, Glass TR, Clarke SP, et al. Rationing of nursing care and its relationship to patient outcomes: the Swiss extension of the International Hospital Outcomes Study. Int J Qual Health Care 2008;20:227-37.

68. Australian Commission on Safety and Quality in Health Care. Patientcentred care: improving quality and safety through partnerships with patients and consumers. Sydney, Australia: ACSQHC, 2011. https:// www.safetyandquality.gov.au/wp-content/uploads/2012/03/PCC_ Paper_August.pdf. (accessed 8 Oct 2018).

69. Koren MJ. Person-centered care for nursing home residents: the culture-change movement. Health Aff 2010;29:312-7.

70. National Health and Medical Research Council. National statement on ethical conduct in human research. Canberra, Australia: The National Health and Medical Research Council, the Australian Research Council, Universities Australia, 2007. https://www.nhmrc. gov.au/guidelines-publications/e72. (accessed 8 Oct 2018). 